\title{
Integrating Measured Force Feedback in Passive Multilateral Teleoperation.
}

\author{
Michael Panzirsch, Thomas Hulin, Jordi Artigas, Christian Ott, Manuel \\ Ferre
}

\begin{abstract}
In teleoperation systems, the master robot receives force feedback from the remote slave side. Thus, the human operator can per- ceive the contact between the slave robot and its environment. Appli- cation of a force sensor at the slave robot improves the performance of the telepresence system in terms of transparency. Still, so far no approach allowing measured force feedback in time delayed multilateral systems that allow the interaction of multiple agents can be found in lit- erature. To this end, this paper presents a multilateral setup with passive measured force feedback based on the time domain passivity approach. Besides this solution to measured force feedback in multilateral systems, the presented approach promises improvements compared to other time invariant and model based approaches for measured force feedback also when applied to bilateral systems. Experiments are presented to allow for a performance analysis of the proposed system design.
\end{abstract}

Keywords: teleoperation, measured force feedback, passivity, TDPA

\section{Introduction}

The enhancement of robot technology in the past few years increased the quality of teleoperation systems that couple a slave robot with a master input device by a controller. Besides former application in space and the nuclear industry, new markets, e.g. in medicine and industrial maintenance evolved. Impedance controlled light weight robots, higher computational performance and modern control techniques improved the transparency of teleoperation systems, i.e. the quality of immersion into the slave's environment that the human operator perceives via his/ her interaction device.

The focus of the present work lies on teleoperation systems that incorporate measurement of the slave contact forces in its environment For instance, in the classical Position-Force measured architecture $\left(P F_{\text {meas }}\right)$ a position $P$ or velocity respectively is sent from master to slaveand a measured force $F_{\text {meas }}$ from slave 
to master through the communication channel $[3,4,7]$. Measured force feedback eases the remote control of the slave's motion in free environment as the slave robot's dynamics are completely masked and only the interaction force with the environment is perceived by the operator. Furthermore, forces of higher bandwidth are transmitted in the $P F_{\text {meas }}$ architecture compared to the PositionForce computed $\left(P F_{\text {comp }}\right)$ architecture. In this type of architecture the force of the PI controller is fed back to the master which can have a damping effect during free motion, i.e. transparency is reduced. Tavakoli et al. studied in [12] the benefits of a 4-Channel architecture that consists of two controllers on each side of the communication channel and feedback of the measured human and environmental interaction forces. In theory, a perfectly transparent system can be achieved through this architecture.

The motivation of this paper originates from multilateral systems that are currently of high interest for training and cooperative scenarios [10]. In particular, multilateral control approaches that consider time delay are based on the principle of passivity. Willaert et al. [14] showed that bilateral control structures which contain force sensors are non-passive. Passivity could be established by representing the system created by the slave robot and the environment through a 1-port network. However, in that approach, the force feedback path is considerably downscaled and a maximmal environment impedance is assumed. In [6], Khademian et al. designed a 4-Channel system for multilateral control. In [8], the Raisbeck passivity criterion was applied. Kanno and Yokokohji [5], Quang and Ryu [11] and Panzirsch et al. [9] presented multilateral teleoperation systems considering time delay. The passivity of the communication channels were guaranteed by the wave variables method and the time domain passivity approach (TDPA) respectively. In [9], a generic approach based on passive modules was developed which serve as the basic multilateral framework for the present work.

In [2], Artigas et al. applied the TDPA in order to handle the effect of time delay in a bilateral $P F_{\text {meas }}$ architecture by representing the system through time domain passivity networks (TDPN). Tobergte and Albu-Schaeffer [13] implemented a full state feedback controller for bilateral teleoperation of a surgical robot. The approach allowed stable interaction with hard and soft environments. The TDPA guaranteed passivity of the overall bilateral teleoperator. In contrast to the present work $[2,13]$ applied the TDPA to the time delay in the communication channel.

The main contribution of this paper is the enhancement of passivity based nonlinear multilateral teleoperation via a new control approach guaranteeing passivity despite use of measured force feedback. Note that this approach is not meant to overcome the effects of time delay. Still, the presented framework can be combined with passivity-based methods tackling time delay like wave variables or time domain control methods. The novel method does not rely on imprecise model parameters and as, furthermore, the passivity is enforced by an additional variable damper in time domain, no conservative controller parametrization is necessary. Thus, the proposed approach also brings benefit when applied in bilateral systems. 
Section 2 provides an overview of our modular multilateral architecture [9] and the related challenges of measured force feedback. The model-free control approach for measured force feedback architectures as the main contribution of the paper is presented in Sect.3. Experiments are presented in Sect. 4 and Sect. 5 summarizes the results.

\section{Multilateral Structure}

The signal flow diagram of a $1 \mathrm{DoF}$ (Degree of Freedom) bilateral $P F_{\text {meas }}$ architecture is depicted in Fig. 1. The human operator is controlling a slave robot in a remote or unaccessi-

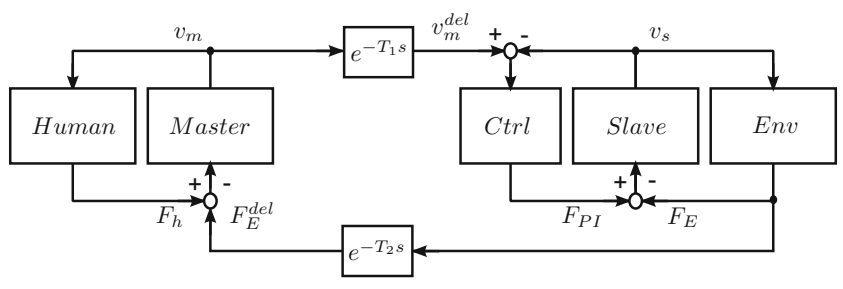

Fig. 1. Signal flow for measured force feedback architecture ble environment (Env) through a master input device. The slave's position is controlled by a position/velocity controller ( $\mathrm{Ctrl}$ ) - acting as a virtual spring - to match the master's position. The measured interaction force between slave and environment is fed back to the master. The communication channel is represented by the Laplace transformation of a pure constant delay $e^{-T_{1} s}$ and $e^{-T_{2} s}$. A virtual damping can be added as a proportional part in the controller $(\mathrm{Ctrl})$ or as local dampers at master and slave devices.

Anderson proposed in [1] the application of the network representation to the analysis of teleoperation systems. The network representation divides a system into several n-ports that are connected by power-conjugated ports. Port $\mathrm{i}$ is an interface of flow $v_{i}$ and effort $F_{i}$ such that a power $P_{i}(t)=F_{i}(t) v_{i}(t)$ can be defined at port i.

Figure 2 shows an exemplary network representation of a $P F_{\text {meas }}$ architecture without time delay. The generalized multilateral system developed in [9] can be analyzed in Fig. 3. The human operator at the master and the slave in its environment are represented as agent subsystems conjointly. Those are connected

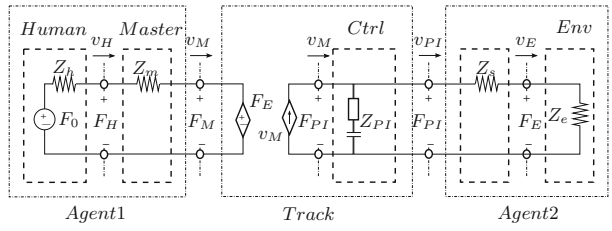

Fig. 2. Network representation of a $P F_{\text {comp }}$ architecture without time delay

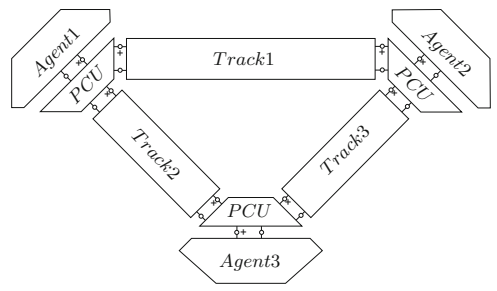

Fig. 3. Network representation of a multilateral teleoperation system 
via power control units (PCU) and tracks containing e.g. controller and communication channel. Figure 4 presents the bilateral network representation for the 1-port passivity approach presented in [14]. This approach for passive measured force feedback cannot be straightforwardly applied to multilateral systems as no 1-port teleoperation subsystem integrating the devices, controllers and the environment can be defined for the multilateral network. This problem is the main motivation for the present work.

\section{Control Design}

Another approach has to be investigated for multilateral systems with measured force feedback. The TDPA for a passive $P F_{\text {meas }}$ module is suggested as a solution and designed in the following. Figure 5 can be considered as a generalization of a bilateral 2-channel teleoperation system (the subsystems

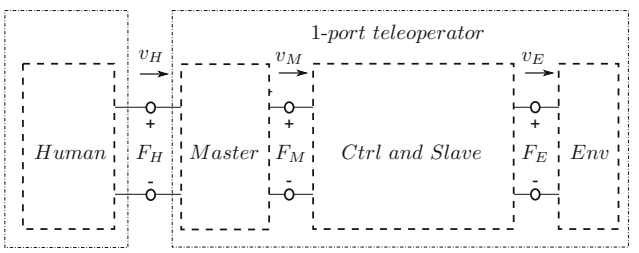

Fig. 4. Network representation of the 1-port teleoperator proposed in [14] $P C_{L / R}$ have to be neglected first).

The setup is split up into two channels representing the two directions of energy flow (compare [2]). Energy sent from master to slave is flowing from left to right (L2R) and the energy sent from slave to master flows in right to left (R2L) direction. In R2L direction a dependent effort source $F_{F B}$ injects energy that is sent from slave to master. The dependent flow source $v_{1}$ injects the energy introduced by the master in L2R direction. The power flowing in the R2L part of the track in direction to the slave is dissipated by the effort source and the power flowing in direction to the flow source in the L2R part of track is dissipated by the flow source. The power at the ports flowing in the different directions can be easily distinguished:

$$
P_{8 b}^{L 2 R}(t)=\left\{\begin{array}{cc}
0, & \text { if } P_{8 b}(t)<0 \\
P_{8 b}(t), & \text { if } P_{8 b}(t)>0
\end{array} \quad \text { and } \quad P_{8 b}^{R 2 L}(t)=\left\{\begin{array}{cl}
0, & \text { if } P_{8 b}(t)>0 \\
-P_{8 b}(t), & \text { if } P_{8 b}(t)<0
\end{array}\right.\right.
$$

with $P_{8 b}(t)=v_{8 b}(t) F_{8 b}(t)$. The same computation of power flow holds for all other ports.

In the case of open loop teleoperation where the master receives no feedback the dependent effort source does not inject energy, as $F_{F B}$ is zero. The system is stable, as the L2R can be proven to be passive. The PI-controller acting as spring and damper is passive and the terminations (i.e. the environment, human operator and their representative effort and flow source) are generally assumed to behave passive in their interactions.

In a $P F_{\text {comp }}$ architecture (compare Fig. 2) $F_{F B}$ is equal to $F_{8 b}$. Every network subsystem is passive in that case. 


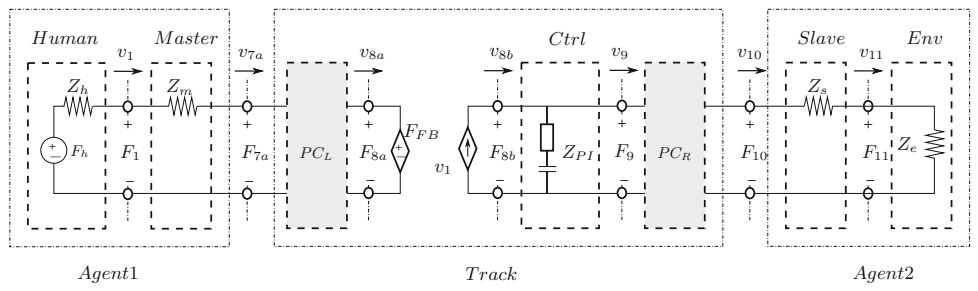

Fig. 5. Network representation of passive $P F_{\text {meas }}$ architecture without time delay

The effort source depends on $F_{11}$ (i.e. the measured force $F_{E}$ in the slave environment) in a $P F_{\text {meas }}$ architecture. Willaert et al. [14] showed that then passivity can only be guaranteed if the 1-port including every subsystem despite the human operator (see Fig. 4) is considered in a frequency-based analysis. Since this frequency-based passivity approach results in a conservative non-adaptive gain parametrization and is not applicable to multilateral systems, time domain control is applied to guarantee passivity of the measured force feedback. The concept is based on the following observations: The system is passive for the $P F_{\text {comp }}$ architecture as the PI has a passive behavior:

$$
\int_{0}^{t} P_{8 b}^{L 2 R}(\tau)+P_{9}^{R 2 L}(\tau) d \tau \geq \int_{0}^{t} P_{8 b}^{R 2 L}(\tau)+P_{9}^{L 2 R}(\tau) d \tau .
$$

At port $8 \mathrm{~b}$ and $8 \mathrm{a}$ the same power is flowing in the $P F_{\text {comp }}$ architecture.

Therefore, as long as the measured force feedback $\left(F_{F B}=F_{11}\right)$ does not inject more energy via the effort source in R2L direction compared to the computed force feedback $\left(F_{F B}=F_{8 b}\right)$, the control architecture remains passive also for $P F_{\text {meas }}$ architecture. The L2R part of the track is not influenced by the measured force feedback.

The resulting concept can be realized by the following implementation:

The energy injected into the PI controller has to be observed and stored.

The energy leaving the PI controller to the slave at port 10 (compare Fig. 5 neglecting PC subsystems) and the energy sent to the master by the independent force source $F_{F B}$ have to be limited depending on the energy storage of the PI controller. This can be solved by dissipative impedance type passivity controllers $P C_{L}$ and $P C_{R}$. Note that impedance type PCs don't cause position drift.

When a power should leave at port 8 a or $9\left(P_{8 a}^{R 2 L}\right.$ or $\left.P_{9}^{L 2 R}\right)$ it has to be checked if enough energy has entered the controller beforehand. The energy content of the controller can be computed (see Fig. 5) in each time step:

$$
\Delta E_{C}(k)=\Delta E_{C}(k-1)+P_{8 b}^{L 2 R}(k)+P_{9}^{R 2 L}(k) .
$$

At first the desired output $P_{\text {out }}^{\text {dem }}$ in both direction of energy flow has to be calculated:

$$
P_{\text {out }}^{\text {dem }}(k)=P_{8 a}^{R 2 L}(k)+P_{9}^{L 2 R}(k) .
$$


If this power $P_{\text {out }}^{\text {dem }}$ is smaller than $\Delta E_{C}$, this power may exit. The PCs are only active if excess power needs to be dissipated:

$$
\begin{aligned}
& P_{\text {diss }}^{P C_{L}}(k)=\left\{\begin{array}{cl}
\left(\Delta E_{C}(k) P_{8 a}^{R 2 L}(k)\right) /\left(P_{\text {out }}^{\text {dem }}(k) T_{s}\right), & \text { if } \Delta E_{C}(k)<P_{\text {out }}^{\text {dem }}(k) T_{s} \\
0, & \text { if } \Delta E_{C}(k)>P_{\text {out }}^{\text {dem }}(k) T_{s}
\end{array},\right. \\
& P_{\text {diss }}^{P C_{R}}(k)=\left\{\begin{array}{cl}
\left(\Delta E_{C}(k) P_{9}^{L 2 R}(k)\right) /\left(P_{\text {out }}^{\text {dem }}(k) T_{s}\right), & \text { if } \Delta E_{C}(k)<P_{\text {out }}^{\text {dem }}(k) T_{s} \\
0, & \text { if } \Delta E_{C}(k)>P_{\text {out }}^{\text {dem }}(k) T_{s}
\end{array}\right.
\end{aligned}
$$

with the system sample time $T_{s}$. The impedance type $P C_{L}$ e.g. dissipates the power $P_{\text {diss }}^{P C_{L}}$ with the variable damping $\alpha_{P C_{L}}$ via the force $F_{P C_{L}}$ reducing the measured force feedback force $\left(F_{7 a}=F_{F B}+F_{P C_{L}}\right)$ :

$$
F_{P C_{L}}(k)=\alpha_{P C_{L}}(k) v_{7 a}(k), \quad \text { with } \quad \alpha_{P C_{L}}(k)=-P_{d i s s}^{P C_{L}}(k) / v_{7 a}^{2}(k) .
$$

The track in Fig. 5 is passive, if the 4-port of $P C_{L}, P I$ and $P C_{R}$ is passive:

$$
E_{7 a}^{10}(k)=\sum_{0}^{k}\left(P_{8 b}^{L 2 R}(k)+P_{9}^{R 2 L}(k)-P_{7 a}^{R 2 L}(k)-P_{10}^{L 2 R}(k)\right) \geq 0 .
$$

This holds, since the power at those 4 ports are monotonously increasing and the passivity controllers assure that the output energy is lower or equal to the input energy:

$$
\begin{gathered}
\int_{0}^{t} P_{8 b}^{L 2 R}(\tau)+P_{9}^{R 2 L}(\tau) d \tau \geq \int_{0}^{t} P_{8 b}^{R 2 L}(\tau)+P_{9}^{L 2 R}(\tau) d \tau \\
P_{7 a}^{R 2 L}(t) \leq P_{8 a}^{R 2 L}(t) \quad \text { and } \quad P_{10}^{L 2 R}(t) \leq P_{9}^{L 2 R}(t)
\end{gathered}
$$

Through this design the biggest benefits of measured force feedback are maintained: If there's no contact with the environment (free motion) the operator's motion will not be hindered by a force, as desired. When the operator steers the slave into a collision, power is flowing from master to slave. Therefore $P C_{L}$ will not vary the force feedback to the master such that the dynamics of the impact can be well perceived.

The measured force feedback will only be varied if the environment injects energy. E.g. in case of an external impact on the slave robot the force feedback may be affected by the $P C_{L}$ as there can be an excess energy output at port $8 a$. The dissipation in the PI controller determines how high this effect is.

The delay free setup of Fig. 5 can be combined with the approach presented in [2], in which the time delay in the communication channel is represented by two Time Domain Power Networks (TDPN, Fig. 6). The energy generated by the time delay in L2R direction e.g. can be observed at port 7a and 5a of TDPN1. The passivity controllers PC1 and PC2 terminating the TDPNs dissipate the energy generated by the time delay and thus guarantee passivity of the communication channel. The resulting track of Fig. 6 can be straightforwardly applied to the multilateral systems proposed in $[9,10]$. 


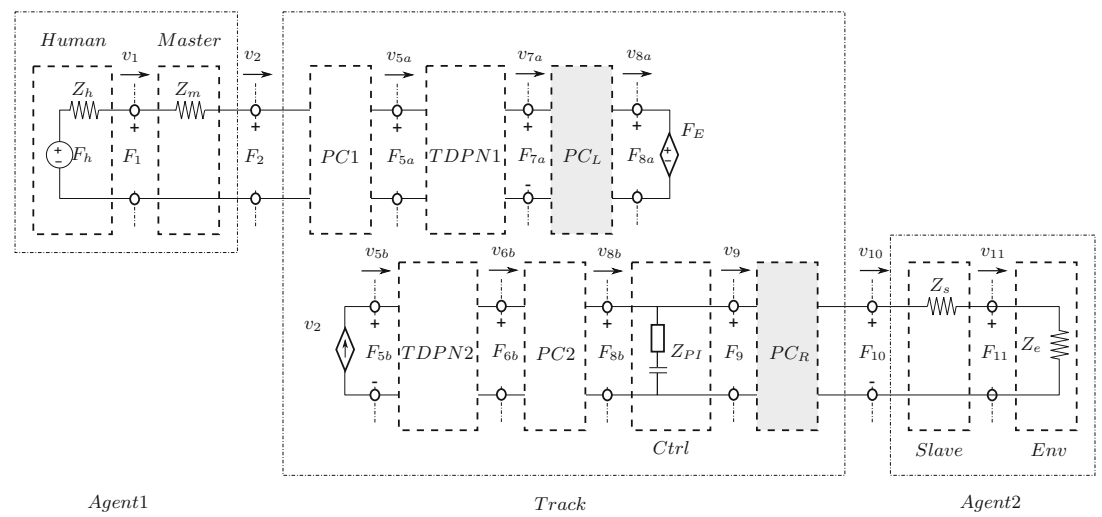

Fig. 6. Network representation of passive $P F_{\text {meas }}$ architecture with time delay

\section{Experiments}

The following experiments were performed with three 1DoF rotatory devices (see Fig. 7) which are equipped with torque sensors in the rotatory center of the grip. The control software was implemented in Matlab/Simulink and running on a QNX-machine in real-time with $1 \mathrm{kHz}$ sampling rate. At first the performance of teleoperation with measured force feedback is compared with computed force feedback in free motion and during a wall contact. The later experiments consider a time delay in the communi-

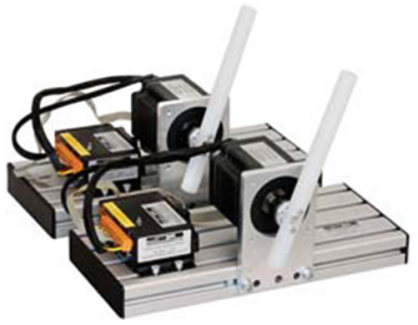

Fig. 7. 1DoF master-slavesystem (courtesy of sensodrive) cation channel and a multilateral setup. A local damping was applied to each device, but in order to test the most critical case for the approach, the damping in the PI controllers of the tracks was set to zero.

In the first experiments the master is controlling the slave at different speeds with computed (see Fig. 8) and measured force feedback (see Fig. 9). During free motion power is flowing mainly from master to slave (compare $P_{9}^{L 2 R}$ in Fig. 8). Comparing Figs. 8 and 9, it is obvious that during low speeds (Fig. 8: $2.5 \mathrm{~s}-4 \mathrm{~s}$; Fig. 9: $4.2 \mathrm{~s}-5.6 \mathrm{~s}$ ), the master receives a higher feedback force when computed force feedback is active. Figure 9 shows that the passivity controllers $P C_{L / R}$ do not need to dissipate energy though the dissipative damping in the controller was set to zero. $E_{7 a}^{10}$ - the sum of input and output energy measured between port $7 a$ and port 10 - is never negative which proofs that the subsystem consisting of $P I, P C_{L}$ and $P C_{R}$ behaves passive. During faster motion (Fig. 8: $1.5 \mathrm{~s}-2.5 \mathrm{~s}$; Fig. 9: $3 \mathrm{~s}-4 \mathrm{~s}$ ) the grip mass leads to a measured feedback force due to high acceleration of the tool mass. The position tracking is satisfactory in all experiments and at all speeds. In the next part of the experiment the operator moves the slave device such that it contacts a wall. Figure 8 shows that energy 

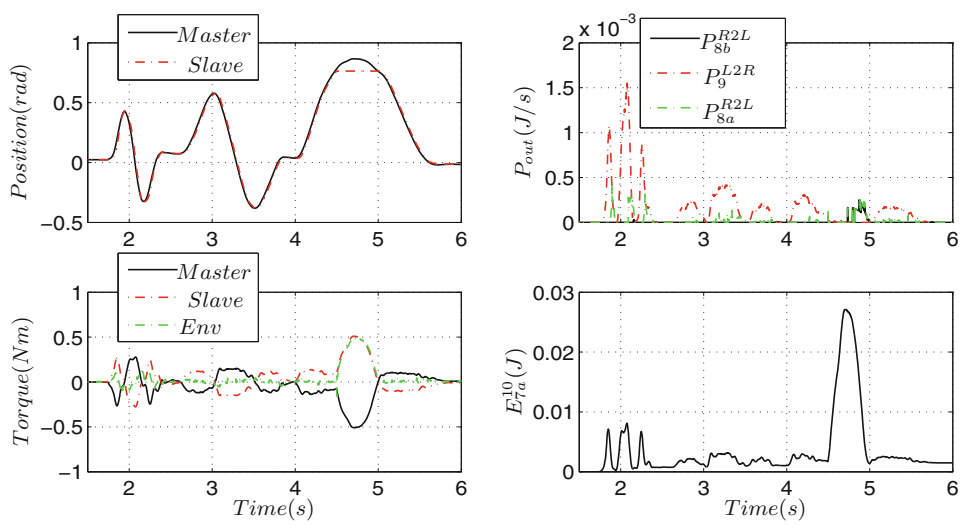

Fig. 8. Free motion and wall contact with computed force feedback

flows from slave to master when the master leaves out of the penetrated wall $(4.8 \mathrm{~s}-5 \mathrm{~s})$. This amount of energy was injected before when the master moved into the wall $(4.4 \mathrm{~s}-4.8 \mathrm{~s})$. As the slave velocity is zero at that time, no energy is leaving to the slave. Figure 9 shows a torque peak $(6.4 \mathrm{~s})$ measured by the sensor during the impact into the wall. This torque is fed back unaltered to the master device. It is obvious that other approaches that demand a constant high down-scaling of the feedback force would result in a worse perception of the environment for the operator in this situation.
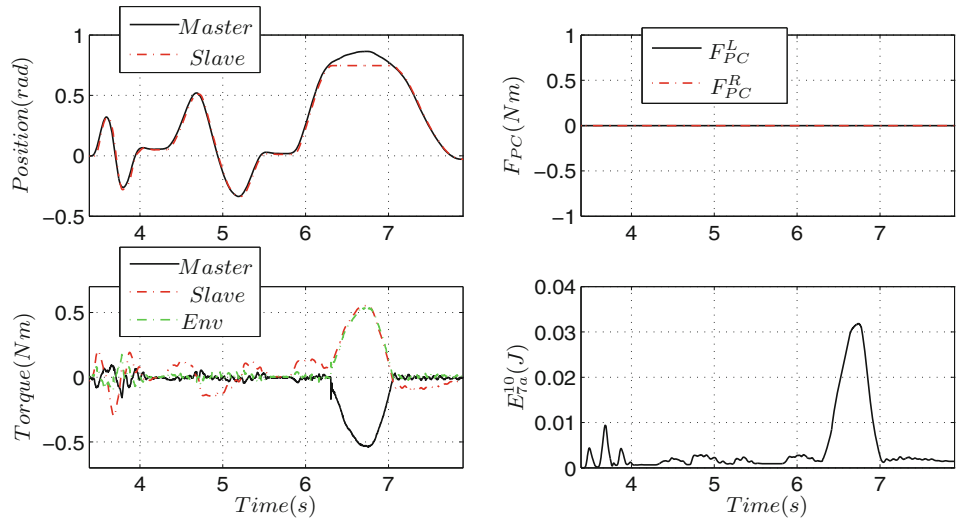

Fig. 9. Free motion and wall contact with measured force feedback

The following experiment considers time delay (compare Fig. 6). Figure 10 depicts the behavior under symmetric $100 \mathrm{~ms}$ roundtrip delay. Comparing Figs. 9 and 10 it can be seen that the position following is of course better in the 

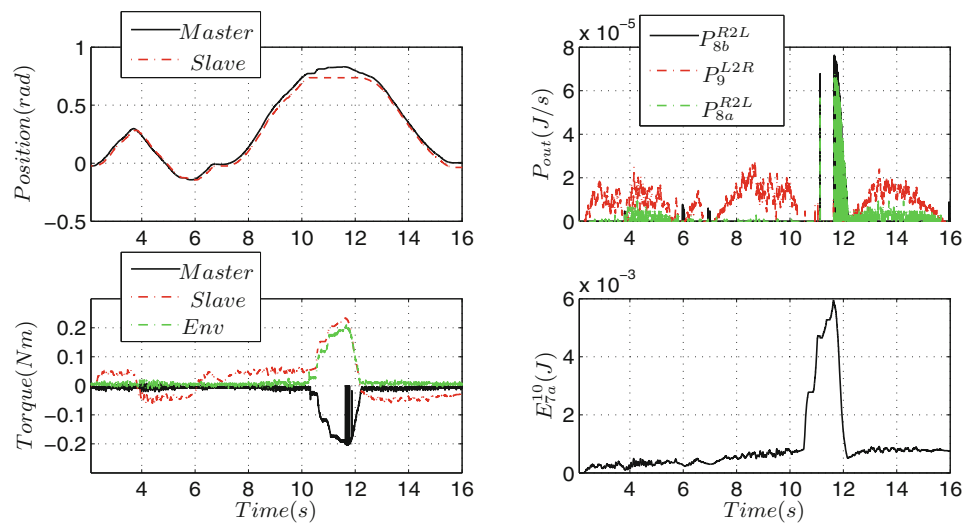

Fig. 10. Wall contact with measured force feedback at $100 \mathrm{~ms}$ roundtrip delay

experiment without delay. $E_{7 a}^{10}$ (Fig. 10) is always positive, i.e. the subsystem consisting of $P I, P C_{L}$ and $P C_{R}$ is still passive.
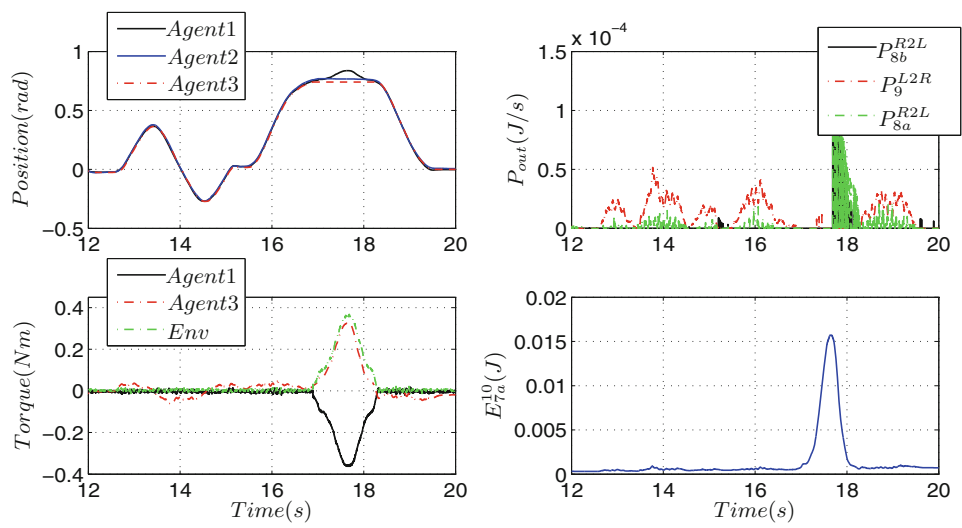

Fig. 11. Position tracking in a multilateral setup (forces and energy behaviour of track2)

A multilateral system as designed in Fig. 3 can be analyzed in Fig. 11. The Agent1 and Agent2 - grasped by the operator's hands - receive measured force feedback from Agent3 (track2,3) whereas Agent1 and Agent2 receive computed force feedback from each other (track1). Note that the plot considers for the sake of clarity only track2. Agent1 has the authority and steers the Agent3 against a wall. The position tracking of the three devices is satisfactory. The energy plot proofs passivity of the subnetwork of concern. 


\section{Conclusion}

A passive module for $P F_{\text {meas }}$ architecture based on the TDPA has been designed for multilateral architectures in the presented work. It could be shown that the system is not conservative as it is designed in the time domain. As other approaches aiming at absolute stability or passivity of measured force feedback systems consider physical model parameters and therefore limit the force feedback more gravely and as the PCs dissipate rarely, the proposed approach can be assumed to provide better performance also for general bilateral systems. Furthermore, the module can be used in combination with other passivity based approaches as e.g. the wave variables method. Experiments with time delay proved the system's adequacy for the classical teleoperation tasks. Subjectively rated, the performance with respect to transparency could be improved substantially in free motion and at fast collisions compared to a $P F_{\text {comp }}$ architecture. In future work the presented approach will be extended to a 4-Channel architecture.

\section{References}

1. Anderson, R.J., Spong, M.W.: Asymptotic stability for force reflecting teleoperators with time delay. Int. J. Robot. Res. 11(2), 135-149 (1992)

2. Artigas, J., Ryu, J.H., Preusche, C., Hirzinger, G.: Network representation and passivity of delayed teleoperation systems. In: IEEE International Conference on Intelligent Robots and Systems (IROS), pp. 177-183 (2011)

3. Daniel, R., McAree, P.: Fundamental limits of performance for force reflecting teleoperation. Int. J. Robot. Res. 17(8), 811-830 (1998)

4. Hannaford, B., Anderson, R.: Experimental and simulation studies of hard contact in force reflecting teleoperation. In: Proceedings of 1988 IEEE International Conference on Robotics and Automation, pp. 584-589. IEEE (1988)

5. Kanno, T., Yokokohji, Y.: Multilateral teleoperation control over time-delayed computer networks using wave variables. In: Haptics Symposium, pp. 125-131 (2012)

6. Khademian, B., Hashtrudi-Zaad, K.: A four-channel multilateral shared control architecture for dual-user teleoperation systems. In: IEEE International Conference on Intelligent Robots and Systems, pp. 2660-2666 (2007)

7. Lawrence, D.A.: Stability and transparency in bilateral teleoperation. IEEE Trans. Robot. Autom. 9(5), 624-637 (1993)

8. Mendez, V., Tavakoli, M.: A passivity criterion for n-port multilateral haptic systems. In: 2010 49th IEEE Conference on Decision and Control (CDC), pp. 274-279 (2010)

9. Panzirsch, M., Artigas, J., Ryu, J.H., Ferre, M.: Multilateral control for delayed teleoperation. IEEE International Conference on Advanced Robotics, pp. 1-6 (2013)

10. Panzirsch, M., Balachandran, R., Artigas, J.: Cartesian task allocation for cooperative, multilateral teleoperation under time delay. In: IEEE International Conference on Robotics and Automation, pp. 312-317 (2015)

11. Quang, H.V., Ryu, J.H.: Stable multilateral teleoperation with time domain passivity approach. In: IEEE International Conference on Intelligent Robots And Systems, pp. 5890-5895 (2013) 
12. Tavakoli, M., Aziminejad, A., Patel, R., Moallem, M.: Enhanced transparency in haptics-based master-slave systems. In: IEEE American Control Conference (ACC), pp. 1455-1460 (2007)

13. Tobergte, A., Albu-Schaeffer, A.: Direct force reflecting teleoperation with a flexible joint robot. In: IEEE International Conference on Robotics and Automation, pp. 4280-4287 (2012)

14. Willaert, B., Corteville, B., Reynaerts, D., Brussel, H.V., Poorten, E.B.V.: Bounded environment passivity of the classical position-force teleoperation controller. In: IEEE International Conference on Intelligent Robots and Systems, pp. 4622-4628 (2009) 